\title{
Two-Dimensional AOA Estimation Based on a Constant Modulus Algorithm
}

\author{
Saleh O. Al-Jazzar, Zoubir Hamici, and Sami Aldalahmeh
}

Al-Zaytoonah University of Jordan, Amman, Jordan

Correspondence should be addressed to Saleh O. Al-Jazzar; saleh.g@zuj.edu.jo

Received 29 June 2016; Revised 11 December 2016; Accepted 25 December 2016; Published 18 January 2017

Academic Editor: Giuseppe Mazzarella

Copyright ( 2017 Saleh O. Al-Jazzar et al. This is an open access article distributed under the Creative Commons Attribution License, which permits unrestricted use, distribution, and reproduction in any medium, provided the original work is properly cited.

\begin{abstract}
We propose a two-dimensional (2D) angle of arrival (AOA) estimator using the algebraic constant modulus algorithm (ACMA). This algorithm was originally introduced to estimate the one-dimensional (1D) AOA. An extension to estimate and automatically pair the elevation and azimuth angles for different sources is derived and proved in this paper. The ACMA method factorises a matrix into two different matrices; one is of constant modulus and contains the azimuth AOA information; however the second was previously ignored. In this paper we will prove that this second matrix contains the elevation AOA information. Thus, $2 \mathrm{D}$ AOA estimation is proved possible using the ACMA method. Simulation results are presented to illustrate the proposed method's performances under different conditions.
\end{abstract}

\section{Introduction}

Direction of arrival denotes the direction from which a propagating wave is impinging on an antenna array, while the angle of arrival estimates this direction by measuring the difference of phases or time delays at the individual elements of the array using the wavenumber. Angle of arrival (AOA) estimation has gained great attention due to its importance in enhancing the received signal quality and/or locating wireless transmitters. Many estimators were developed in the literature to estimate the AOAs. Initially, these methods were developed to estimate one-dimensional AOAs. Different antenna array structures were assumed for the AOA estimation such as the uniform linear antenna array (ULA) and circular antenna array structures.

In many practical applications it is required to estimate both the elevation and azimuth AOAs. So, many methods were developed initially to estimate the elevation and azimuth angles separately using two ULAs (such as $L$-shaped or parallel ULAs). Then, another method was applied to pair up these estimated elevation and azimuth angles [1-7]. Other methods were also developed in which the elevation and azimuth angles were estimated jointly without the need for any pairing technique; these methods require the use of two ULAs [1, 8-11]. As depicted by Figure 1, for the $L$-shaped ULAs, one ULA will be placed on one axis ( $x$-axis) and the other will be placed on a perpendicular axis ( $z$-axis).

In this paper we present an elevation and azimuth AOA estimator based on the algebraic constant modulus algorithm (ACMA) presented in [12]. The process is performed blindly at the receiver without the need for any training. The ACMA method, upon which the 2D AOA estimator is based, performs factorisation on a received signal block to retrieve the constant modulus (CM) part contained in this block. This is valid under the assumption that the original transmitted signal has a CM feature.

A cross-correlation matrix is initially formed from the antenna model; then the ACMA algorithm is applied on it to get two matrices; one is $\mathrm{CM}$ and contains the azimuth AOAs information for different sources and the second, previously ignored, holds their corresponding elevation AOAs. Thus, a 2D AOA estimator can be achieved by utilising both matrices of the ACMA factorisation. Also, automatic pairing between different elevation and azimuth AOAs is achieved for different sources. The proposed estimator does not require 


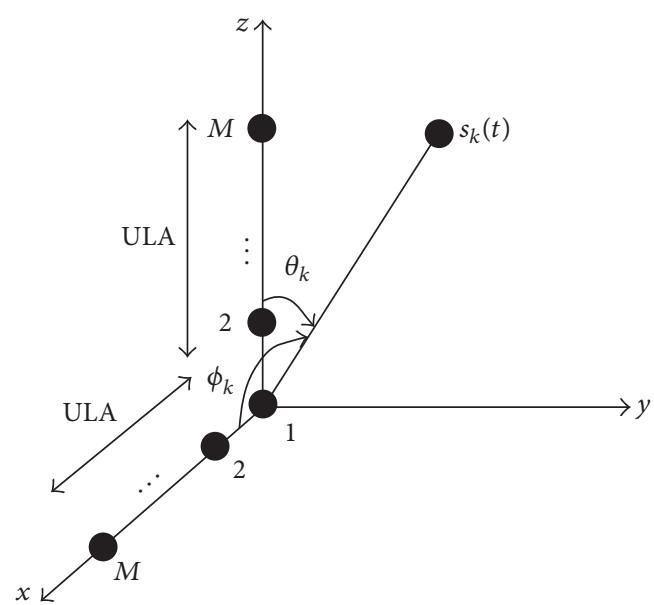

FIGURE 1: Layout for the $L$-shaped antenna array.

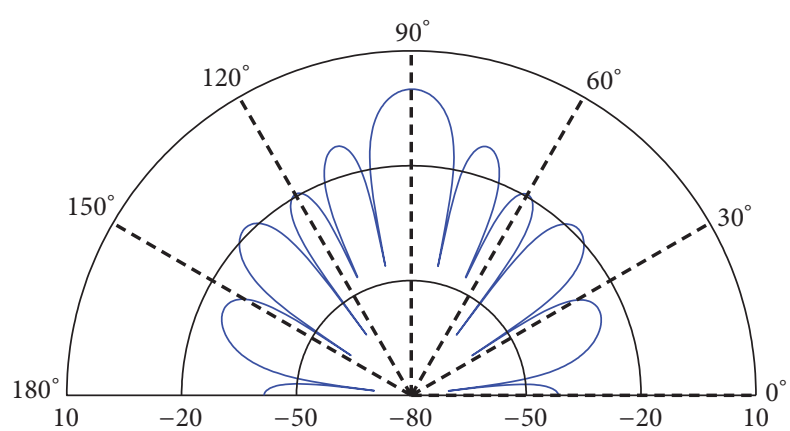

FIGURE 2: Radiation pattern for 1D linear antenna array presenting 10 nulls plotted in $\mathrm{dB}$ versus $\mathrm{AOA}$ in degrees, with 20 elements and interspacing $d=0.12$, with a side lobe level of $-13 \mathrm{~dB}$.

any training, so it is considered a blind estimator. The proposed estimator is named the 2D ACMA based algorithm.

\section{Problem Formulation}

In this section, we present the narrowband received signal model that will be utilised for joint 2D AOA estimation. The $L$-shaped antenna array has two arrays in two perpendicular axes (Figure 1). The radiation pattern for one array is illustrated by Figure 2 . Assume $K$ sources signals, $\left\{\check{s}_{k}(t)\right\}_{k=1}^{K}$, impinging upon the array. The signal $\check{s}_{k}(t)$ is represented as $\check{s}_{k}(t)=$ $s_{k}(t) \cos \left(2 \pi f_{c} t\right)=\mathfrak{R}\left\{s_{k}(t) \exp \left(j 2 \pi f_{c} t\right)\right\}$, where $f_{c}$ is the carrier frequency, and $s_{k}(t)=\sum_{i} \alpha_{k}(i) g(t-i T)$, where $i$ is an integer which represents the time index, and $s_{k}(t)$ is the low-pass equivalent of $\check{s}_{k}(t), T$ is the symbol period, $\alpha_{k}(i)=$ $A_{k} \beta_{k}(i)$, where $\beta_{k}(i) \in(-1,+1)$ which represents the symbol parity for a BPSK signal, and $A_{k}$ is a positive constant which represents the amplitude of $s_{k}(t)$, and $g(t)$ is the sampled (raised cosine) pulse shaping function with

$$
g(n T)= \begin{cases}1, & \text { if } n=0 \\ 0, & \text { otherwise }\end{cases}
$$

where $n$ is an integer so that $s_{k}(n T)=\alpha_{k}(n)$.
Since we are looking at $2 \mathrm{D}$ AOA estimation, the $k$ th source signal arriving at the array will have an elevation angle, $\theta_{k}$, and an azimuth angle, $\phi_{k}$. Because of the $L$-shaped antenna orientation shown in Figure 1, the angle $\phi_{k}$ is taken between the $x$-axis and the source signal arrival direction, and the angle $\theta_{k}$ is taken between the $z$-axis and the source signal arrival direction. We use the superscript notation \{\} to denote the estimated value of a variable (for example, $\widehat{h}$ is the estimated value of $h$ ). So, to start developing the received signal model, let us first consider the sampled received signal at the antenna elements (after the matched filter stage), from $m=1$ to $m=M$, that are located in the $z$-axis direction, and let us call it $\mathbf{r}_{z}(i)$. This is represented as

$$
\mathbf{r}_{z}(i)=\sum_{k=1}^{K} \alpha_{k}(i) \mathbf{a}\left(\theta_{k}\right)+\mathbf{n}_{z}(i)
$$

where $\mathbf{a}\left(\theta_{k}\right)=\left[\begin{array}{lll}a_{1}\left(\theta_{k}\right) & \cdots & a_{M}\left(\theta_{k}\right)\end{array}\right]^{T}$ and

$$
a_{m}\left(\theta_{k}\right)=\exp \left(j \frac{2 \pi}{\lambda} d \times(m-1) \cos \left(\theta_{k}\right)\right)
$$

with $\lambda$ being the signal wavelength and $d$ being the distance between consecutive antenna elements. In addition, $\mathbf{n}_{z}(i)$ is the noise vector added to the received signal at the antenna elements (from $m=1 \rightarrow M$ ) located along the $z$-axis and it is additive white Gaussian noise (AWGN) with a covariance matrix of $\sigma^{2} \mathbf{I}_{M \times M}$, where $\mathbf{I}_{M \times M}$ is the $M \times M$ identity matrix. Finally, $(\cdot)^{T}$ represents the transpose operator.

Similarly, the sampled received signal at the antenna elements, from $m=1$ to $m=M$, that are located in the $x$-axis direction, is given the notation $\mathbf{r}_{x}(i)$ and is represented as

$$
\mathbf{r}_{x}(i)=\sum_{k=1}^{K} \alpha_{k} \mathbf{b}\left(\phi_{k}\right)+\mathbf{n}_{x}(i)
$$

where $\mathbf{b}\left(\phi_{k}\right)=\left[\begin{array}{lll}b_{1}\left(\phi_{k}\right) & \cdots & b_{M}\left(\phi_{k}\right)\end{array}\right]^{T}$ and

$$
b_{m}\left(\phi_{k}\right)=\exp \left(j \frac{2 \pi}{\lambda} d \times(m-1) \cos \left(\phi_{k}\right)\right) .
$$

Likewise, $\mathbf{n}_{x}(i)$ is the AWGN noise vector added to the received signal at the antenna elements (from $m=1 \rightarrow M$ ) located along the $x$-axis, and it also has a covariance matrix of $\sigma^{2} \mathbf{I}_{M \times M}$. So, both $M \times 1$ received signal vectors can be written in matrix form as

$$
\begin{aligned}
& \mathbf{r}_{z}(i)=\mathbf{A}(\boldsymbol{\theta}) \boldsymbol{\alpha}(i)+\mathbf{n}_{z}(i), \\
& \mathbf{r}_{x}(i)=\mathbf{B}(\boldsymbol{\phi}) \boldsymbol{\alpha}(i)+\mathbf{n}_{x}(i),
\end{aligned}
$$

where

$$
\begin{aligned}
\mathbf{A}(\boldsymbol{\theta}) & =\left[\begin{array}{lll}
\mathbf{a}\left(\theta_{1}\right) & \cdots & \mathbf{a}\left(\theta_{K}\right)
\end{array}\right], \\
\mathbf{B}(\boldsymbol{\phi}) & =\left[\begin{array}{lll}
\mathbf{b}\left(\phi_{1}\right) & \cdots & \mathbf{b}\left(\phi_{K}\right)
\end{array}\right], \\
\boldsymbol{\alpha}(i) & =\left[\begin{array}{lll}
\alpha_{1}(i) & \cdots & \alpha_{K}(i)
\end{array}\right] .
\end{aligned}
$$

From now on we will drop the index $i$ from $\mathbf{r}_{z}(i)$ and $\mathbf{r}_{x}(i)$ for simplicity. 
Now, we formulate a cross-correlation matrix between the signal received at the array on $z$-axis and the signal received at the array on $x$-axis; that is, we consider the following crosscorrelation matrix:

$$
\begin{aligned}
& \mathbf{R}_{z x}=E\left[\mathbf{r}_{z} \mathbf{r}_{x}^{H}\right], \\
& \mathbf{R}_{z x}=\mathbf{A}(\boldsymbol{\theta}) \mathbf{P}_{s} \mathbf{B}(\phi)^{H},
\end{aligned}
$$

where

$$
\mathbf{P}_{s}:=E\left[\boldsymbol{\alpha} \boldsymbol{\alpha}^{H}\right]=\operatorname{diag}\left(\left[\begin{array}{lllll}
p_{1} & \cdots & p_{k} & \cdots & p_{K}
\end{array}\right]\right)
$$

with $p_{k}$ being the power of the $k$ th source. This formation of the cross-correlation matrix is the matrix upon which the ACMA algorithm will be applied. Next, we give a description on the ACMA method which will be used in the 2D AOA estimation.

2.1. The ACMA Algorithm. Given a block of data $\mathbf{X}=$ $\left[\begin{array}{llll}\mathbf{x}_{1} & \mathbf{x}_{2} & \cdots & \mathbf{x}_{N}\end{array}\right]$ of size $M \times N$, where

$$
\mathbf{X}=\mathbf{Q S}
$$

with $\mathbf{S}$ being the matrix composed of constant modulus elements and $\mathbf{Q}$ being some full rank matrix. Then, the aim of the ACMA method is to factorise $\mathbf{X}$ to get back $\widehat{\mathbf{Q}}$ and $\widehat{\mathbf{S}}$ or alternatively find a beamformer matrix $\mathbf{W}$, where $\widehat{\mathbf{S}}=\mathbf{W}^{H} \mathbf{X}$ with some phase ambiguity.

The ACMA described in [12] starts by a prewhitening step (which is not considered in the original ACMA method proposed in [13]). To do so, we compute $\widehat{\mathbf{R}}_{x}=(1 / N) \sum_{n} \mathbf{x}_{n} \mathbf{x}_{n}^{H}$. Then, take its eigenvalue decomposition (EVD); that is, $\widehat{\mathbf{R}}_{x}=$ $\widehat{\mathbf{U}} \widehat{\boldsymbol{\Sigma}}^{2} \widehat{\mathbf{U}}^{H}$. Set the prewhitening matrix as $\mathbf{F}=\widehat{\mathbf{U}}_{s} \widehat{\boldsymbol{\Sigma}}_{s}^{-1}$, where $\widehat{\Sigma}_{s}$ is a diagonal matrix which contains the square root of the $D$ dominant eigenvalues and $\widehat{\mathbf{U}}_{s}$ are their corresponding eigenvectors. Also, $D$ represents the number of sources. Now, prefilter the data, $\underline{\mathbf{x}}_{n}=\mathbf{F}^{H} \mathbf{x}_{n}$. A whitening transformation or Principal Components Analysis (PCA) is a dimensionality reduction algorithm that transforms a vector of random variables with a known correlation matrix into a set of new variables whose correlation is the identity matrix which means that such signals are uncorrelated. This prewhitening step will reduce the matrix size over which the ACMA algorithm is performed from $M \times N$ to $D \times N$. Recall that $D=K$ is the number of sources which is assumed to be smaller than $M$ (the number of antenna elements). Now, we will define the matrix $\mathbf{T}$ which is similar to the beamformer matrix $\mathbf{W}$ but in the whitened domain; that is, $\mathbf{T}=\mathbf{F}^{H} \mathbf{W}$.

Next, form the matrix

$$
\widehat{\widehat{\mathbf{C}}}=\frac{1}{N} \sum_{n}\left(\underline{\overline{\mathbf{x}}}_{n} \otimes \underline{\mathbf{x}}_{n}\right)\left(\underline{\overline{\mathbf{x}}}_{n} \otimes \underline{\mathbf{x}}_{n}\right)^{H}-\operatorname{vec}\left\{\underline{\widehat{\mathbf{R}}}_{x}\right\}\left\{\underline{\widehat{\mathbf{R}}}_{x}\right\}^{H},
$$

where $\otimes$ is the Kronecker product and $\{-\}$ is the complex conjugate operator, so, for example, $\bar{h}$ is the complex conjugate of $h$ and $\operatorname{vec}(\mathbf{H})$ is the process of stacking the columns of some matrix $\mathbf{H}$ into a vector. It is proven in [12] that the null space of $\widehat{\widehat{C}}$ spans the same subspace as the columns of the beamformer matrix T. So, we compute the null space of $\underline{\widehat{C}}$ which we will denote as $\mathbf{V}$ to help find $\mathbf{T}$. The source separation beamformer matrix $\mathbf{T}$, in the whitened domain constraint, will be found by an iterative process of joint diagonalisation such that the solutions of the beamformer columns elements are scaled to unit-norm vectors $\left(\left\|\mathbf{t}_{i}\right\|\right)$ as a decorrelation step defined by the properties of the constant modulus algorithm in the presence of noise [12]. So the solution can be written in the form

$$
\mathbf{T}=\arg _{\left\|\mathbf{t}_{i}\right\|, \mathbf{M} \text { is invertable }}\|\mathbf{V M}-\overline{\mathbf{T}} \circ \mathbf{T}\|,
$$

where $\circ$ is the Katri-Rao product.

To satisfy both conditions (the basis of the null space of $\widehat{\mathbf{C}}$ and the structured matrix) a joint diagonalisation process is performed which is transformed into the following iterative process [12].

Start with initializing the matrix $\mathbf{T}$ by setting it to $\mathbf{T}=\mathbf{I}$, where I is the identity matrix. Then, apply the following steps:

$$
\mathbf{M}=\mathbf{V}^{H}(\overline{\mathbf{T}} \circ \mathbf{T}) .
$$

Then, recondition the matrix $\mathbf{M}$ by

$$
\begin{aligned}
& \mathbf{M}^{\prime}=\operatorname{recond}(\mathbf{M}):=\sum_{j} \sigma_{j}^{\prime} \mathbf{u}_{j} \mathbf{v}_{j}^{H}, \\
& \quad \text { where } \sigma_{j}^{\prime}= \begin{cases}1, & \sigma_{j}<0.5 \\
\sigma_{j}, & \sigma_{j} \geq 0.5,\end{cases}
\end{aligned}
$$

where $\sigma_{j}, \mathbf{u}_{j}$, and $\mathbf{v}_{j}$ are the $j$ th singular value, left singular vector, and right singular vector of $\mathbf{M}$. Then project $\mathbf{V}$ on $\mathbf{M}^{\prime}$, as follows:

$$
\mathbf{Y}=\mathbf{V} \mathbf{M}^{\prime}
$$

Now, consider the $i$ th column of $\mathbf{Y}$ and name it $\mathbf{y}_{i}$ and apply the inverse of the vectorisation process; that is, $\mathbf{Y}_{i}=$ $\operatorname{vec}^{-1}\left(\mathbf{y}_{i}\right)$. Then, take the SVD of $\mathbf{Y}_{i}$ and consider the left singular vector corresponding to the largest singular value; that is, let

$$
\mathbf{Y}_{i}=\Sigma_{j} \gamma_{j} \mathbf{p}_{j} \mathbf{q}_{j}^{H}
$$

and set

$$
\mathbf{t}_{i}=\mathbf{p}_{1},
$$

where $\mathbf{t}_{i}$ is the $i$ th column vector of $\mathbf{T}$. The process of joint diagonalisation is repeated until convergence. The described joint diagonalisation process is closely related to the (canonical) polyadic decomposition which is used for factor retrieval, dimensionality reduction, and signal denoising to name but a few. This multiway array (tensor) decomposition is able to take into account, for example, spatial, temporal, and spectral information simultaneously and retrieve factors under mild conditions $[14,15]$.

After estimating $\mathbf{t}_{i}$, for all $i$, thus estimating beamformer matrix in the whitened domain (T), we can set the matrix $\mathbf{W}$ to be

$$
\mathbf{W}=\mathbf{F T}
$$


which is the beamformer matrix in the original (nonwhitened) domain; then $\mathbf{S}$ is estimated as

$$
\widehat{\mathbf{S}}=\mathbf{W}^{H} \mathbf{X} .
$$

After presenting the ACMA method, we can apply it to estimate the 2D AOA by applying it to $\mathbf{R}_{z x}$.

2.2. 2D ACMA Based AOA Estimator. Looking at the definition of the cross-correlation matrix, $\mathbf{R}_{z x}$, in (8), we can see that $\mathbf{B}(\phi)$ is of constant modulus elements. So, we can apply the ACMA method to factorise $\mathbf{R}_{z x}$ (similar to $\mathbf{X}$ in (10) but with size $M \times M$ instead of $M \times N$ ) into the constant modulus matrix $\mathbf{U}^{H} \widehat{\mathbf{B}}(\phi)^{H}$ (similar to $\mathbf{S}$ in (10)) and $\widehat{\boldsymbol{\Psi}}=\widehat{\mathbf{A}}(\theta) \widehat{\mathbf{P}}_{s} \mathbf{U}$ (similar to $\mathbf{Q}$ in (10)), where $\mathbf{U}$ is a diagonal matrix which causes the phase ambiguity in the factorised matrices.

Notice that the elements of $\widehat{\mathbf{B}}(\phi)$ are all of constant modulus with its size of $K \times M$ with $\mathbf{U}$ a diagonal matrix which will not affect the relative phases of each column of $\widehat{\mathbf{B}}(\phi)$ or $\widehat{\mathbf{A}}(\theta)$. Also, notice that the matrix $\widehat{\mathbf{P}}_{s}$ will not affect the complex elements angles in $\widehat{\mathbf{A}}(\theta)$ (because $\widehat{\mathbf{P}}_{s}$ is a real diagonal matrix). Thus, the elevation angles information in $\widehat{\mathbf{A}}(\theta)$ is reserved in $\widehat{\Psi}$ and can be deduced by

$$
\widehat{\theta}_{k}=\cos ^{-1}\left(\frac{\arg \left(\widehat{\psi}_{\mathrm{avg}}\right)}{2 \pi d / \lambda}\right)
$$

with

$$
\widehat{\psi}_{\mathrm{avg}}=\frac{\sum_{m=1}^{M-1}\left(\widehat{\psi}_{m+1}\left(\theta_{k}\right) / \widehat{\psi}_{m}\left(\theta_{k}\right)\right)}{M-1}
$$

where $\widehat{\psi}_{m}\left(\theta_{k}\right)$ is the $k$ th element of the $m$ th column of $\widehat{\boldsymbol{\Psi}}$ and $\widehat{\psi}_{\text {avg }}$ is the average of all relative phases, not affected by phase ambiguity. Similarly, the azimuth angles can be deduced from $\widehat{\mathbf{B}}(\phi)$ by

$$
\widehat{\phi}_{k}=\cos ^{-1}\left(\frac{\arg \left(\widehat{B}_{\mathrm{avg}}\right)}{2 \pi d / \lambda}\right)
$$

with

$$
\widehat{B}_{\mathrm{avg}}=\frac{\sum_{m=1}^{M-1}\left(\widehat{b}_{m+1}\left(\phi_{k}\right) / \widehat{b}_{m}\left(\phi_{k}\right)\right)}{M-1} .
$$

Thus, both elevation and azimuth angles are estimated for each source using this 2D ACMA based algorithm. It is worth mentioning that since matrix $\mathbf{A}(\boldsymbol{\theta})$ is also constant modulus, then we can apply the ACMA algorithm on the cross-correlation matrix $\mathbf{R}_{x z}$ (instead of $\mathbf{R}_{z x}$ ) which is given by

$$
\mathbf{R}_{x z}=\mathbf{R}_{z x}^{H}=\mathbf{B}(\phi) \mathbf{P}_{s} \mathbf{A}(\boldsymbol{\theta})^{H} .
$$

Next, we will provide simulation results for the proposed algorithm.

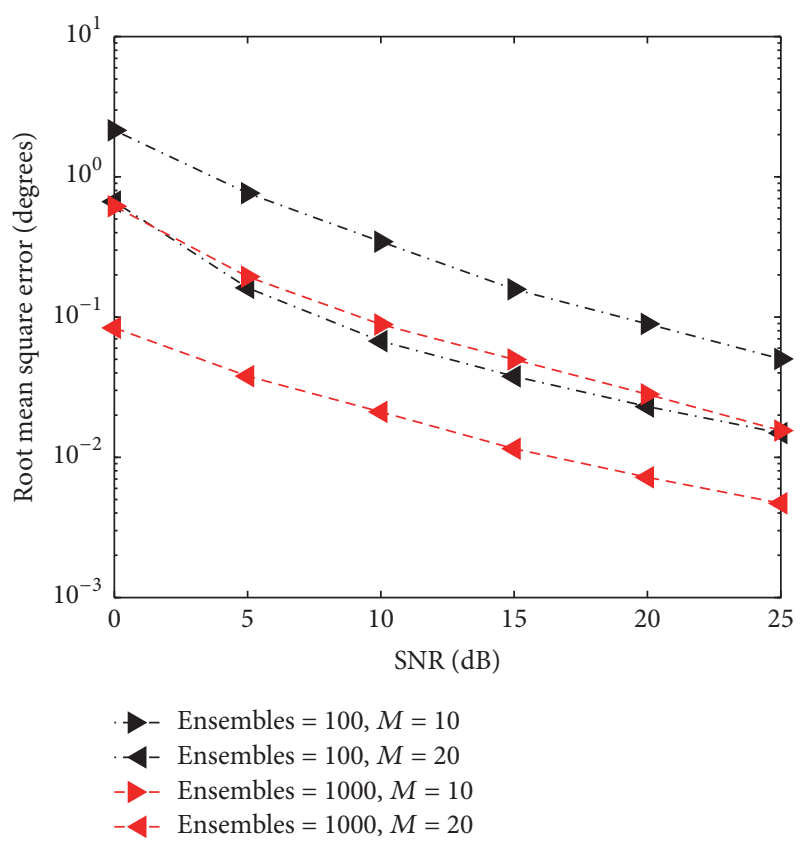

FIgURE 3: Root mean square error in degrees using the 2D ACMA based method versus SNR in $\mathrm{dB}$.

\section{Simulation Results}

Simulations are performed with MATLAB on a dual core computer, i5 processor clocked at $2.5 \mathrm{GHz}$ with a RAM of $6 \mathrm{~GB}$. Results are presented for 2D AOA estimation using a 2D ACMA based algorithm. The number of sources is two with elevation angles $\theta_{1,2}=\left[75^{\circ}, 45^{\circ}\right]$ and azimuth angles $\phi_{1,2}=\left[80^{\circ}, 50^{\circ}\right]$. The number of ensembles over which the simulation results are averaged is 1000 ensembles. The near far ratio (NFR) which is the ratio of power between both sources is set at $-20 \mathrm{~dB}$. Figure 3 shows the root mean square error (RMSE) for the elevation and azimuth AOAs estimation using the 2D ACMA based method versus different signal to noise ratios (SNRs). The number of antenna elements and number of ensembles over which the cross-correlation matrix is averaged are variable as shown in the figure. The results show that the performance is enhanced as the number of antenna elements, ensembles number, and SNR increase.

Figure 4 shows the RMSE for the elevation and azimuth AOAs estimation using the 2D ACMA based method versus different elevation angle difference between the two sources. The elevation angle for the second source is $\theta_{2}=45^{\circ}$ and the first source elevation angle is computed by adding the difference to $\theta_{2}$. The results show that the performance is enhanced as the elevation angle difference increases.

Figure 5 shows the RMSE for the elevation and azimuth AOAs estimation using the 2D ACMA based method versus different NFR ratios between both sources. The results show that the performance is degraded as the NFR ratio increases.

Another simulation is carried out to show the span of AOAs within which the 2D ACMA based algorithm can work. So, Figure 6 illustrates the RMSE for the elevation AOA versus different elevation AOAs for a single source. $M$ and 


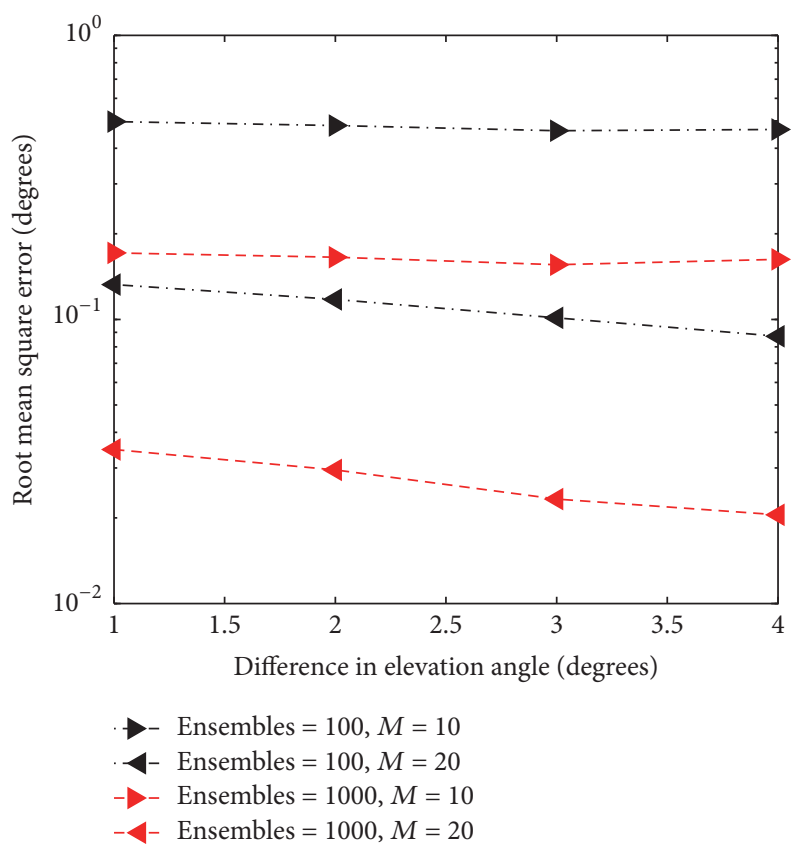

FIGURE 4: Root mean square error in degrees using the 2D ACMA based method versus AOA difference between two sources in degrees.

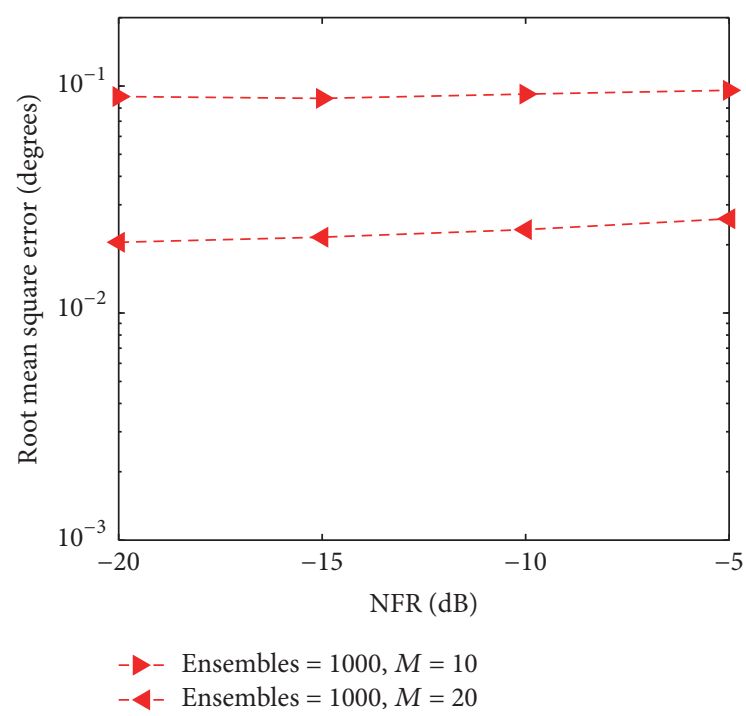

Figure 5: Root mean square error in degrees using the 2D ACMA based method versus NFR in dB.

SNR are set to 10 and $10 \mathrm{~dB}$, respectively. The 2D ACMA based algorithm has a high accuracy in the range $20^{\circ}$ to $160^{\circ}$ with an error less than $0.1^{\circ}$ for 1000 ensembles (lower curve), while it achieves an error of $0.3^{\circ}$ within a range between $35^{\circ}$ and $145^{\circ}$ for 100 ensembles (upper curve). For both results, the azimuth is fixed at $80^{\circ}$. Meanwhile, Figure 7 shows the span behaviour for two values of the antenna size $M$. Lower curve is for $M=20$, while upper curve is for $M=10$. Clearly, the 2D ACMA based performance is enhanced as the size of antenna $M$ increases but the span over which the AOAs are estimated decreases. This latter behaviour illustrates the

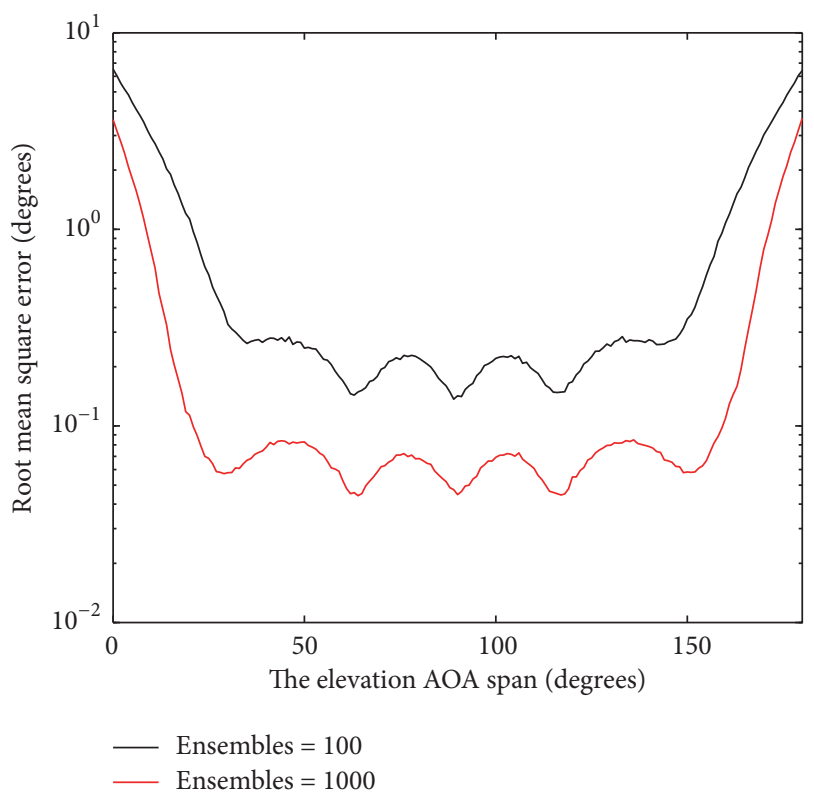

FIGURE 6: Root mean square error in degrees using the 2D ACMA based method versus AOA span in degrees.

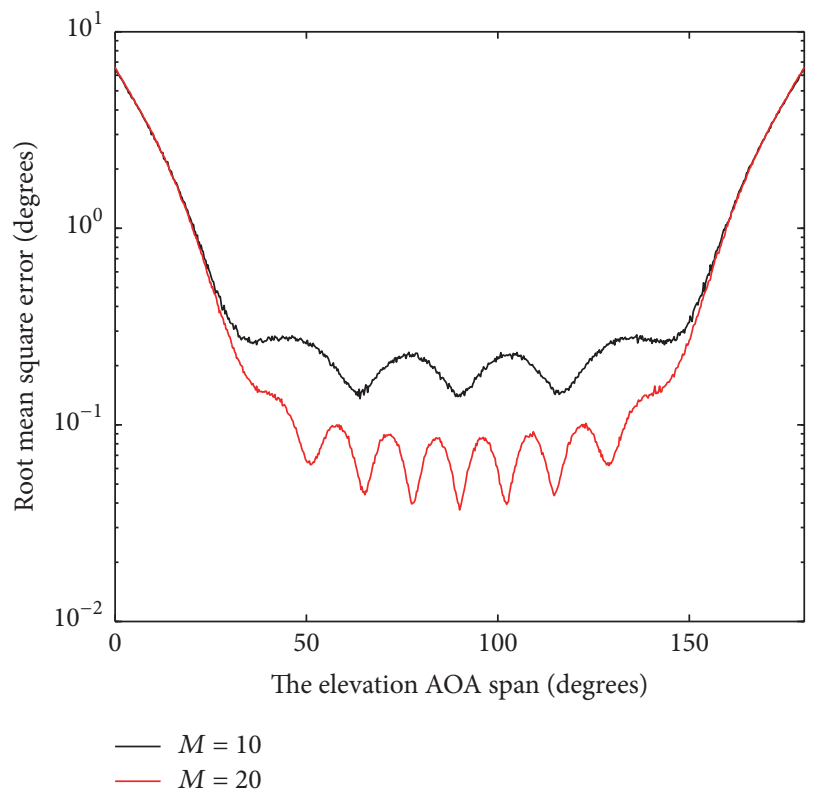

FIGURE 7: Root mean square error in degrees using the 2D ACMA based method versus AOA span in degrees.

compromise between range and accuracy which is expected. The oscillations present in both figures, Figures 6 and 7, are due to the radiation pattern of antenna arrays shown in Figure 2 which is oscillatory by nature.

Figure 8 shows the locus of 10 runs of the 2D ACMA based algorithm where the elevation and azimuth AOAs true values and their corresponding estimations are plotted in the same figure. $M, \mathrm{NFR}$, and SNR are set to $10,-5 \mathrm{~dB}$, and $10 \mathrm{~dB}$, respectively, which shows the high accuracy of the algorithm.

In terms of processing time performance, the time that takes the 2D ACMA based to estimate two pairs of the 


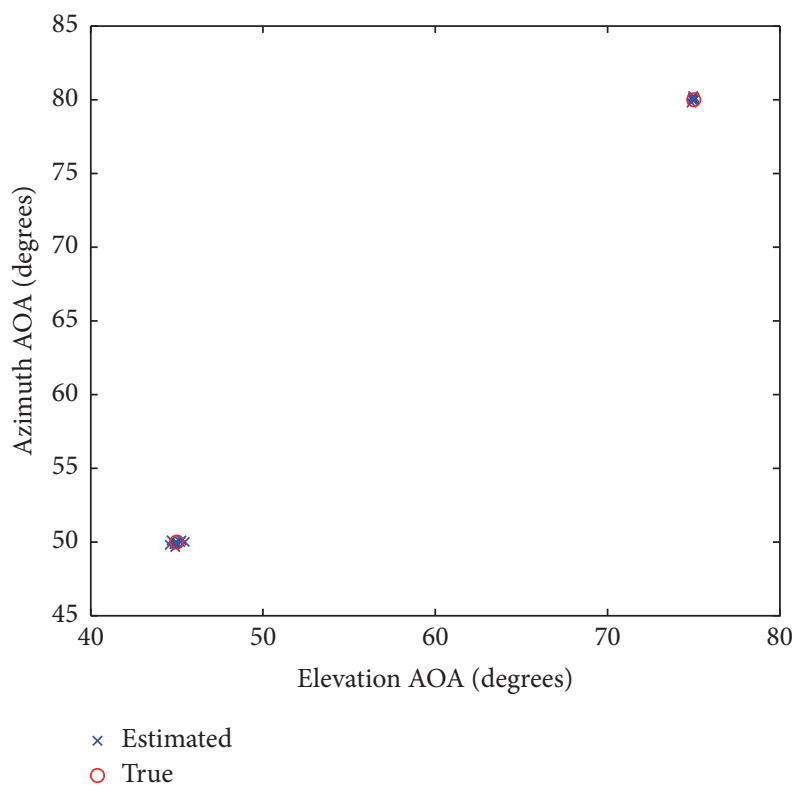

FIGURE 8: Locus of the true and estimated elevation and azimuth AOAs in degrees for 10 different runs.

elevation and azimuth AOAs for two sources, evaluated with MATLAB, is about 8 milliseconds for a number of ensembles equal to 100 .

\section{Conclusion}

In this paper a $2 \mathrm{D} \mathrm{AOA}$ estimator based on the ACMA method is presented. The $2 \mathrm{D}$ extended CM estimator utilises the $L$-shaped structure to form the $2 \mathrm{D}$ antenna array. The model of the antenna array is represented by a crosscorrelation matrix through which the elevation and azimuth angles are estimated simultaneously for different sources. The estimator does not require any training nor it requires any pairing process to combine the elevation and azimuth angles for each source. Simulation results show the effectiveness of the new 2D ACMA based algorithm applied on an $L$-shaped uniform linear arrays. A high accuracy multisource AOAs estimation of $0.1^{\circ}$ is achieved within a wide range of $140^{\circ}$ for both perpendicular antenna arrays.

\section{Competing Interests}

The authors declare that they have no competing interests.

\section{Acknowledgments}

This work is supported by the Dean of Research, AlZaytoonah University of Jordan, under Grant no. 1/25/2014 for Electrical Engineering Research Laboratory, Communication, and Embedded signal Processing Group.

\section{References}

[1] N. Tayem and H. M. Kwon, "L-shape 2-dimensional arrival angle estimation with propagator method," IEEE Transactions on Antennas and Propagation, vol. 53, no. 5, pp. 1622-1630, 2005.

[2] Y. Wu, G. Liao, and H. C. So, "A fast algorithm for 2-D directionof-arrival estimation," Signal Processing, vol. 83, no. 8, pp. 18271831, 2003.

[3] S. Kikuchi, H. Tsuji, and A. Sano, "Pair-matching method for estimating 2-D angle of arrival with a cross-correlation matrix," IEEE Antennas and Wireless Propagation Letters, vol. 5, no. 1, pp. 35-40, 2006.

[4] R. Roy and T. Kailath, "ESPRIT estimation of signal parameters via rotational invariance techniques," Optical Engineering, vol. 29, pp. 296-313, 1990.

[5] M. Haardt and J. A. Nossek, "Unitary ESPRIT: how to obtain increased estimation accuracy with a reduced computational burden," IEEE Transactions on Signal Processing, vol. 43, no. 5, pp. 1232-1242, 1995.

[6] T.-H. Liu and J. M. Mendel, "Azimuth and elevation direction finding using arbitrary array geometries," IEEE Transactions on Signal Processing, vol. 46, no. 7, pp. 2061-2065, 1998.

[7] T. Xia, Y. Zheng, Q. Wan, and X. Wang, "Decoupled estimation of 2-D angles of arrival using two parallel uniform linear arrays," IEEE Transactions on Antennas and Propagation, vol. 55, no. 9, pp. 2627-2632, 2007.

[8] S. O. Al-Jazzar, D. C. McLernon, and M. A. Smadi, "SVD-based joint azimuth/elevation estimation with automatic pairing," Signal Processing, vol. 90, no. 5, pp. 1669-1675, 2010.

[9] J.-J. Jiang, F.-J. Duan, Y.-C. Li, and X.-N. Hua, “Two-dimensional angles estimation method and its improved methods for single source with a sparse array," Progress In Electromagnetics Research C, vol. 46, pp. 41-50, 2014.

[10] L. Wang, Z. Chen, and G. Wang, "High resolution DOA estimation algorithm based on nonuniform L-shaped array," in Proceedings of the International Conference on Advances in Computer Science and Engineering (CSE '13), Los Angeles, Calif, USA, July 2013.

[11] H. Liao, L. Gan, and P. Wei, "A novel 2-D ESPRIT algorithm for 2-D DOA estimation," Journal of Information and Computational Science, vol. 8, no. 10, pp. 1861-1871, 2011.

[12] A.-J. van der Veen, "Asymptotic properties of the algebraic constant modulus algorithm," IEEE Transactions on Signal Processing, vol. 49, no. 8, pp. 1796-1807, 2001.

[13] A.-J. D. van der Veen and A. Paulraj, "An analytical constant modulus algorithm," IEEE Transactions on Signal Processing, vol. 44, no. 5, pp. 1136-1155, 1996.

[14] G. Zhou and A. Cichocki, "Canonical polyadic decomposition based on a single mode blind source separation," IEEE Signal Processing Letters, vol. 19, no. 8, pp. 523-526, 2012.

[15] X. Luciani and L. Albera, "Canonical polyadic decomposition based on joint eigenvalue decomposition," Chemometrics and Intelligent Laboratory Systems, vol. 132, pp. 152-167, 2014. 


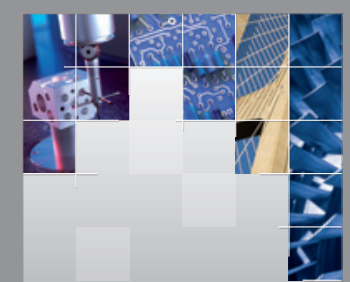

\section{Enfincering}
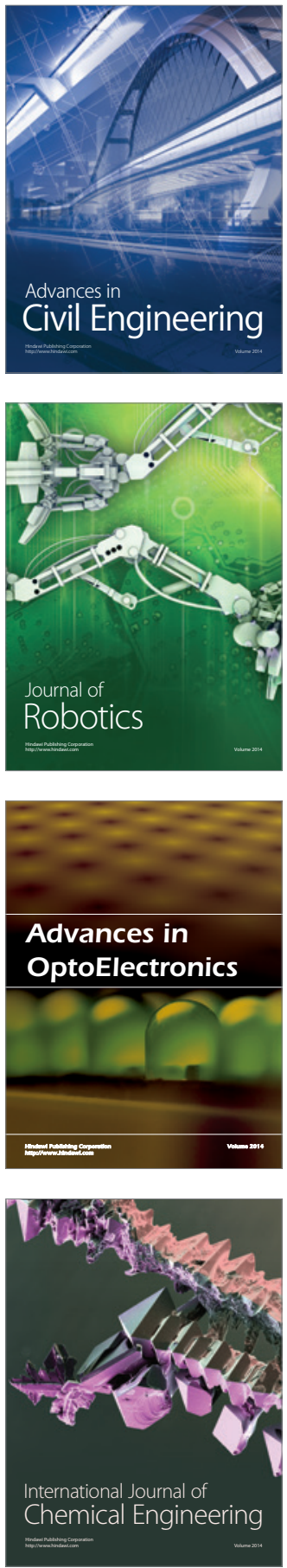

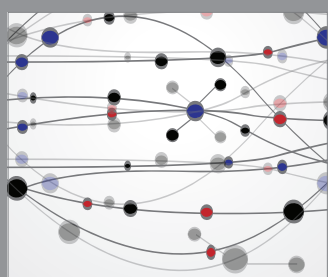

The Scientific World Journal

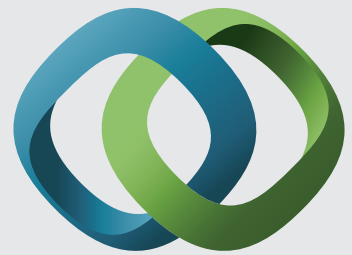

\section{Hindawi}

Submit your manuscripts at

https://www.hindawi.com
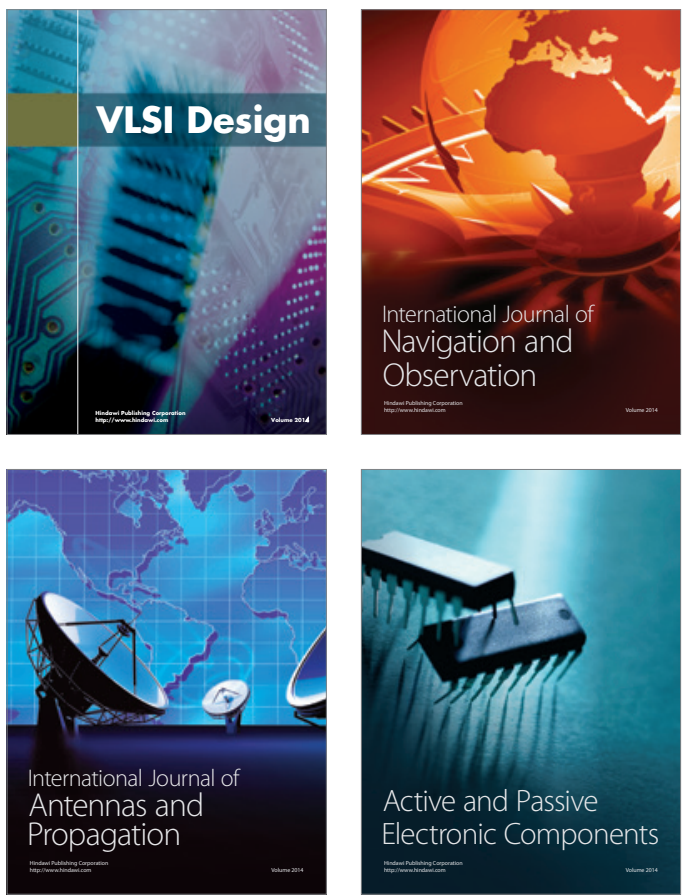
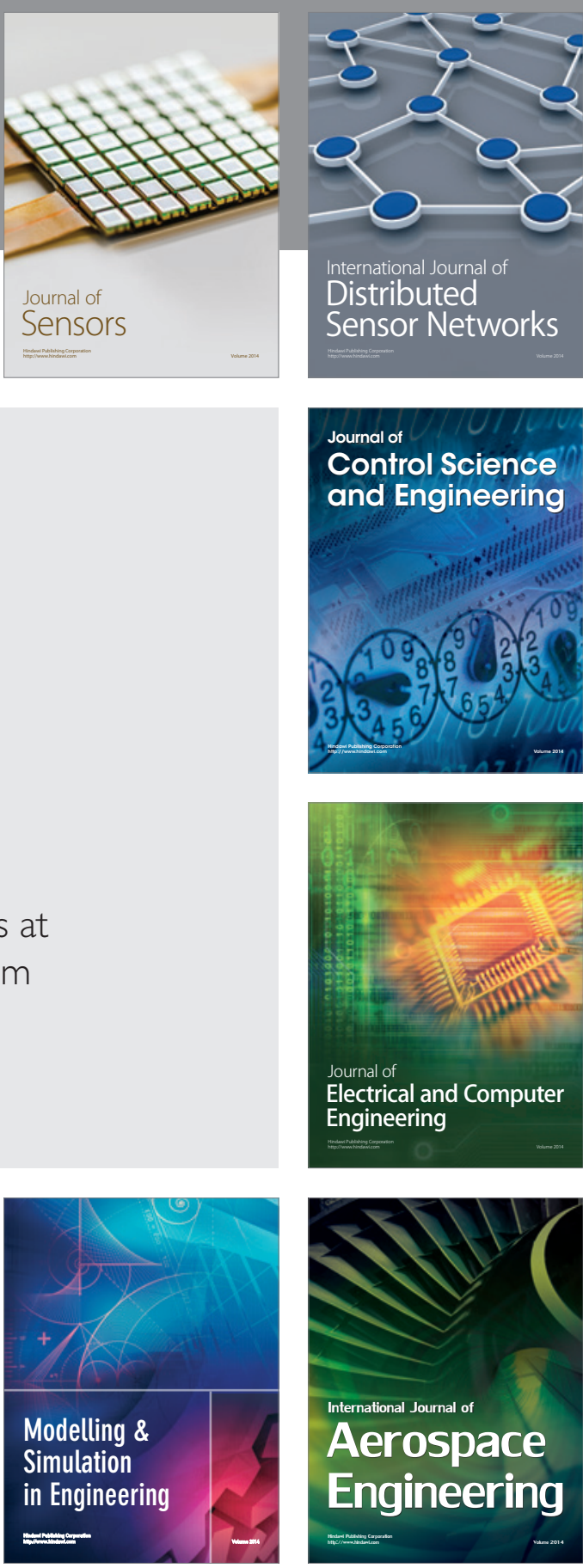

International Journal of

Distributed

Sensor Networks

$-$

Joumal of

Control Science

and Engineering
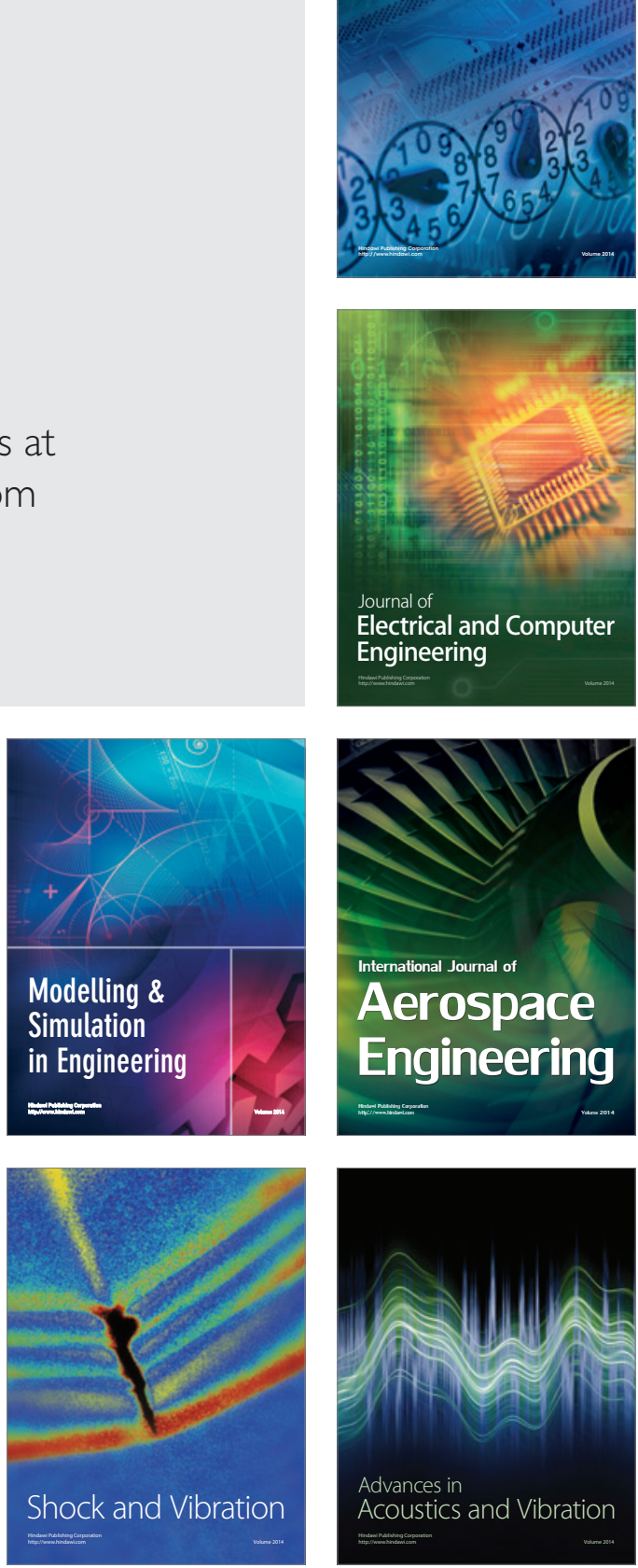